\title{
Spatial and Temporal House Price Diffusion in the Netherlands: A Bayesian Network Approach
}

\author{
Alfred Larm Teye ${ }^{\mathrm{a}, *}$, Daniel Felix Ahelegbey ${ }^{\mathrm{b}}$ \\ ${ }^{a}$ Delft University of Technology, Department of Research for the Built Environment (OTB), The Netherlands \\ ${ }^{b}$ Department of Mathematics and Statistics, Boston University, MA 02215, USA
}

\begin{abstract}
Following the 2007-08 Global Financial Crisis, there have been a growing research interest on the spatial interrelationships between house prices in many countries. This paper examines the spatio-temporal relationship between house prices in the twelve provinces of the Netherlands using a recently proposed econometric modelling technique called Bayesian graphical vector autoregression (BG-VAR). This network approach enables a data driven identification of the most dominant provinces where house price shocks may largely diffuse through the housing market and it is suitable for analysing the complex spatial interactions between house prices. Using temporal house price volatilities for owner-occupied dwellings, the results show evidence of house price diffusion pattern in distinct sub-periods from different provincial housing submarkets in the Netherlands. We observed particularly prior to the crisis, diffusion of temporal house price volatilities from Noord-Holland.
\end{abstract}

Keywords: Graphical models, House price diffusion, Spatial dependence, Spillover effect JEL classification: C11; C15; C32; C52; R20; R32

\section{Introduction}

The collapse of house prices during the 2007-08 Global Financial Crisis (GFC) slowed down economic growth in many countries. After the GFC, researchers and governments alike have been seeking to understand the dynamics of house price development in order to resuscitate the stagnating housing market and the general economy. This has consequently led to a new research agenda that specifically seeks insights into spatial interactions and diffusion between the regional housing markets. House prices vary over space and time, but developments of house prices across regions may not be entirely independent of each other. As explained by Gong et al. (2016), there is significant variations in regional house prices, however, they interrelate spatially over time, and it is therefore paramount for governments to understand this interrelationship in formulating policies to regulate the overall functioning of the housing market.

${ }^{*}$ Corresponding author at: Julianalaan 134, South-Holland, 2628 BL, Delft, The Netherlands. E-mail: a.l.teye@tudelft.nl, Tel: +31628616454 
Spatial interrelationship between regional house prices may take the form of a long-run convergence or a temporal diffusion mechanism. Long-run convergent property markets equilibrate and remain integrated over long period of time (Holmes and Grimes, 2008; Cook, 2005; Cotter et al., 2011). Temporal house price diffusion is also sometimes known in the literature as ripple or spillover effect (see Meen, 1999). This market phenomenon depicts the situation where temporal house price volatility in one region is believed to propagate to house prices in other regions with a transitory or permanent effect (Balcilar et al., 2013; Canarella et al., 2012; Pollakowski and Ray, 1997). Empirical evidence in support of this temporal house price diffusion mechanism exists in the context of the US (Canarella et al., 2012; Holly et al., 2010; Pollakowski and Ray, 1997) and the UK (Meen, 1999, 1996; Holly et al., 2011). More recent results from China and other developing countries also learn support to the house price diffusion hypothesis (see Gong et al., 2016; Lee and Chien, 2011; Nanda and Yeh, 2014; Balcilar et al., 2013). However, in most of these previous studies, the hypothesis is tested for a lead-lag relationship where it is assumed a priori that the diffusion will start from some economically "superior region".

In this paper, we shed light on the spatial and temporal house price diffusion for the case of the Netherlands. The focus is specifically as follows: First, we investigate if there is a spatial dependence of temporal house price volatilities and a diffusion pattern between provinces in the Netherlands. Secondly, we are interested in identifying from the data the province where temporal house price volatilities may predominantly diffuse. Lastly, we investigate if these spatio-temporal relationships vary over time, particularly, if they are unchanged before and after the GFC.

We employ a graphical network approach for studying these spatio-temporal house price dynamics. Graphical modelling is a class of multivariate analysis that uses graphs consisting of nodes and edges to study the interaction and path dependence between variables. The nodes in this graph represent the variables while the edges denote their interactions and dependence structure (see Lauritzen, 1996; Eichler, 2007). The graphical modelling approach has become popular as a more natural way to discover hidden and complex interactions among multiple variables. It is applied mostly in the study of contagion and systemic risk analysis in the financial sector where there is complicated and non-linear relationships between variables (see Ahelegbey, 2016, for a more comprehensive review). Like most financial variables, one indeed expects a complex interrelationships between regional house prices which can easily be handled by the graphical network approach.

In essence, our paper makes four main contributions to the literature. First, we analyse the spatio-temporal house price dynamics in Netherlands which has an entirely different market settings from those studied in the previous papers. The housing market in the Netherlands is unique in many respects. It is a highly regulated market where central and local authorities play an active role. The market is extremely inefficient because of high transaction costs and 
regulated use of building plots (see Vermeulen and Rouwendal, 2007). In general, demand and supply factors in the Dutch owner-occupied housing sector are outcomes of the government housing policies besides income and population growth (see Boelhouwer et al., 2004; Toussaint and Elsinga, 2007). This paper is first to study the spatio-temporal house price dynamics in this unique market setting of the Netherlands and hence adds an interesting dimension to the subject. The second contribution of the paper is that it demonstrates the usefulness of graphical techniques in analysing the spatio-temporal house price dynamics. To the best of our knowledge, this is the first empirical paper that puts forward the graphical framework as an alternative for analysing the house price diffusion mechanism.

The paper specifically uses the recent Bayesian graphical vector autoregression (BG-VAR) model proposed by Ahelegbey et al. (2016a). The BG-VAR is a data driven approach where the directed edges of the network represent causal relationships. This connects to the third contribution of our paper. We estimate spatial interactions that have causal interpretations rather than mere lagged correlations (see details in Ahelegbey et al., 2016a). Fourth, we deduce the central regional market where house price volatilities possibly diffuse from the data. This is contrary to previous studies which assume a priori some "bigger cities" as central in investigating the house price volatility diffusion process (e.g. Holly et al., 2011). This potential selection bias is avoided in our approach because such central region can be easily inferred from the network using statistical measures for the centrality. Moreover, we can learn the diffusion pattern of the temporal house price fluctuations from the network structure without resort to impulse response analysis. ${ }^{1}$ The specific statistical measures for analysing the centrality and diffusion mechanism are made precise in subsequent sections.

Using quarterly data on temporal house price volatilities (1995:Q1-2016:Q1) for existing owner-occupied dwellings from the twelve provinces of the Netherlands, our results support a temporal dependence and diffusion dynamics between the provincial housing markets. These interrelationships however varied over the study period in terms of the degree of dependence and the centrally dominant sub-markets. Noord-Holland in particular was most predominant region where temporal house price volatilities likely diffused to other provinces prior to the crisis. In application, the results of this paper are relevant for policy makers who wish to direct regulations aimed at avoiding temporal regional house price volatilities from cascading systemically to other regions. Likewise, investors may find the results applicable in diversifying and managing risks of their housing portfolios.

We organised the remaining sections of the paper as follows: A brief overview of the related literature is provided in Section 2. Section 3 describes the BG-VAR model. The description of our data is presented in Section 4 while Section 5 discusses the empirical results. The entire paper is concluded in Section 6.

\footnotetext{
${ }^{1}$ The BG-VAR nevertheless also has an underlying variable selection mechanism that will yield a parsimonious VAR model for performing impulse response analysis.
} 


\section{Extant Literature}

Many scholars have been working on the spatio-temporal house price diffusion or the so-called ripple effect and a vast literature now exist. An extensive review of the literature is provided by Balcilar et al. (2013) and most recently by Nanda and Yeh (2014) and Gong et al. (2016). We only provide a brief summary here. The study of this ripple effect hypothesis actually began from the UK when English researchers observed that house prices rise, during an upswing, first from the South-East (mostly London) and then spread out to other parts of the country (Giussani and Hadjimatheou, 1991; Meen, 1996, 1999). According to Pollakowski and Ray (1997) house price diffusion will not necessarily occur between neighbouring housing markets, but may require some form of economic interrelationship. Meen (1999) likewise shared the view of Pollakowski and Ray (1997), and noted that spatial dependence may not be necessary for explaining the ripple effect. Meen (1999) then suggested four probable mechanisms through which rising house prices from one region may later manifest in other parts of the UK. These channels according to the author include: migration, equity transfer, spatial arbitrage and spatial patterns in house price determinants. As also noted later by Canarella et al. (2012), migration particularly may lead to house price ripple effect if households relocate in response to changes in the spatial distribution in house prices.

Meen (1999) methodologically assumed that regional house prices will react to shocks at different rates, and then went on to provide an empirical framework for testing the ripple effects. This was effectively equivalent to testing the stationarity of the regional to national house price ratios. Although Meen (1999) was unsuccessful in confirming the ripple effect with the augmented Dickey-Fuller test of Dickey and Fuller (1979), the author's empirical framework became the basis for other scholars who later found empirical evidence using more sophisticated stationarity test procedures. Cook (2003), for instance adopted the threshold and momentum threshold autoregressive test procedures while Holmes and Grimes (2008) used a combination of unit root test and principal component analysis to confirm the spillover effect in the UK. Canarella et al. (2012) similarly studied the house price diffusion effect in the US by using a combination of the generalised least squares (GLS) version of the DickeyFuller, non-linear unit root tests and other test procedures that control for structure breaks. Balcilar et al. (2013) also adopted a Bayesian and non-linear unit root tests, with and without structure breaks to investigate the ripple effect in the South African housing market. The panel seemingly unrelated regressions augmented Dickey-Fuller (SURADF) has equally been employed by other scholars (e.g. Lee and Chien, 2011; Holmes, 2007).

Recently, tremendous effort has also been channelled, relying on the advancement in the econometric literature, in refining the methodology for testing the ripple effect hypothesis beside the "Meen framework". Holly et al. (2011), for example proposed a dynamic modelling approach where they allow shocks from the dominant region to propagate to other regions and then echo back. The authors found support for the ripple effect using this approach for the UK 
with London as the dominant region. Gong et al. (2016) also adopted similar method in their study of ripple effect for 10 regions in the Pan-Pearl river of China. Nanda and Yeh (2014), in a related study also suggested using a dynamic panel-spatial model. Some researches equally advocated formulating a vector autoregressive (VAR) model and subsequently testing for Granger Causality (GC) and/or performing (generalised) impulse response analysis (IRA) to examine the ripple effect hypothesis. Brady (2014), for example captured the spatial diffusion of regional housing prices in the US with impulse response functions estimated directly from a single equation spatial autoregressive model. Vansteenkiste and Hiebert (2011) used a global VAR model to study if there are house price spillovers across the euro area countries. Gupta and Miller (2012a) and Gupta and Miller (2012b), similarly formulated different VAR models after which they tested for GC and performed IRA to verify the spatial diffusion phenomenon for various Metropolitan Statistical Areas (MSA) in the US.

The approach in this paper also starts with a baseline VAR model. It follows Ahelegbey et al. (2016a) which converts a VAR model to a Bayesian graphical network (BG-VAR). The BG-VAR identifies temporal and directional dependency between variables and it is somewhat related to the concept of GC. The GC, however adopts a pairwise (or conditional pairwise) analysis to identify the dependence patterns without accounting for the structural uncertainties. On the other hand, the BG-VAR employs a Bayesian technique which incorporates necessary prior information to explore the structure and to apply model averaging. Ahelegbey (2016) provided an empirical evidence that support the superior efficiency of the BG-VAR over the GC in producing dependence patterns that are more suitable to capture complex interdependencies. Investigating the dependence structure between multiple time series with the BG-VAR model is generally more convenient for researchers and policy makers to understand directional or causal relationships. The graphical component especially aids a visual understanding of the interactions between the time series.

\section{The Bayesian Graphical Vector Autoregressive (BG-VAR) Model}

This section presents the formulation of the BG-VAR model adopted in this paper. Assume for a moment that temporal house price volatilities in one region is a result of earlier shock to house prices in other regions. We can formulate a vector autoregressive process of order $p(\operatorname{VAR}(p))$ to capture these interdependencies. As mentioned earlier, some authors study the spatial and temporal house price dynamics by testing for Granger causality (GC) and performing impulse response analysis from this underlying VAR model.

Let $Y_{t}$ denote the vector of house price volatilities at the time $t$ from $n$ regions. We can write the $\operatorname{VAR}(p)$ process for $Y_{t}$ following the equation

$$
Y_{t}=\sum_{i=1}^{p} B_{i} Y_{t-i}+u_{t}=B X_{t}+u_{t}, \quad u_{t} \sim \mathcal{N}\left(\mathbf{0}, \Sigma_{u}\right)
$$


where $t=p+1, \ldots, T ; p$ is the maximum lag order; $B_{i}, 1 \leq i \leq p$ is $n \times n$ matrix of coefficients; $B=\left(B_{1}, \ldots, B_{p}\right)$ is $n \times n p$ and $X_{t}=\left(Y_{t-1}^{\prime}, \ldots, Y_{t-p}^{\prime}\right)^{\prime}$ is $n p \times 1$ stacked autoregressive coefficient matrices and lags of the house price volatilities respectively. The set of equations in (1) captures the structure of the interactions between the regional house price volatilities and Ahelegbey et al. (2016a) show that the temporal dependencies between them could be inferred from $B$. For example when the volatility of house prices in one region depends only on a subset but not on earlier shock to house prices in all the regions, there are components of $B$ that become zero. In general, $B_{i j}$ measures the anticipated effect of changes in the $j$-th predictor $\left(X_{j, t}\right)$ on the house price development in the $i$-th region $\left(Y_{i, t}\right)$.

Ahelegbey et al. (2016a) show that the VAR model (1) can be operationalised as a graphical model using the relation $B=(G \circ \Phi)$, where $G$ is a binary $(0 / 1)$ matrix, $\Phi$ is a coefficients matrix, both of dimension $n \times n p$, and (o) is the element-by-element product. The elements of $G$ represent the presence or absence of an edge (interaction) between volatility of house prices in pairs of regions. A one-to-one correspondence between $B$ and $\Phi$ conditional on $G$ can be identified. That is, $B_{i j}=\Phi_{i j} \neq 0$, if $G_{i j}=1$; and $B_{i j}=0$, if $G_{i j}=0$.

As an example, consider an arbitrary five-dimensional $\operatorname{VAR}(1)$ with coefficients matrix

$$
B=\left(\begin{array}{ccccc}
\beta_{11} & 0 & 0 & 0 & 0 \\
\beta_{21} & 0 & \beta_{23} & 0 & 0 \\
\beta_{31} & 0 & \beta_{33} & 0 & 0 \\
0 & 0 & \beta_{43} & \beta_{44} & 0 \\
0 & \beta_{52} & 0 & 0 & \beta_{55}
\end{array}\right)
$$

where the non-zero elements of $B$ are real numbers. The network that depicts the temporal dependence among the variables associated with (2) can be visualised in Figure 1. The links in the network indicate lagged dependencies between the variables without self lag effects (selfloops). Since $\beta_{21} \neq 0, Y_{1, t-1}$ has a significant impact on $Y_{2, t}$, which we denote as $Y_{1} \rightarrow Y_{2}$.

\begin{tabular}{|c|c|c|c|c|c|}
\hline & $Y_{1, t-1}$ & $Y_{2, t-1}$ & $Y_{3, t-1}$ & $Y_{4, t-1}$ & $Y_{5, t-1}$ \\
\hline$Y_{1, t}$ & 1 & 0 & 0 & 0 & 0 \\
\hline$Y_{2, t}$ & 1 & 0 & 1 & 0 & 0 \\
\hline$Y_{3, t}$ & 1 & 0 & 1 & 0 & 0 \\
\hline$r_{4, t}$ & 0 & 0 & 1 & 1 & 0 \\
\hline$Y_{5, t}$ & 0 & 1 & 0 & 0 & 1 \\
\hline
\end{tabular}

(a)

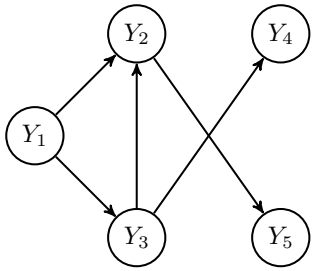

(b)

Figure 1: Network matrix and diagram associated with the temporal dependence in the fivedimensional VAR(1) process in (2). Note: links are lagged effects without self-loops.

For the analysis in this paper, it suffices to estimate only the network structure captured by $G$. Let $D_{t}=\left(X_{t}^{\prime}, Y_{t}^{\prime}\right)^{\prime}$ be a $d \times 1$ vector, where $d=n+n p$ and assume $D_{t} \sim \mathcal{N}\left(\mathbf{0}, \Omega^{-1}\right)$, where $\Omega$ is a $d \times d$ precision matrix. The joint distribution for all the variables in $D_{t}$ can be 
summarised with a graphical model and represented by the pair $(G, \Omega) \in(\mathcal{G} \times \Theta)$. Here, $G$ is a directed acyclic graph (DAG) of the relationships among the variables in $D_{t}, \Omega$ consists of the VAR model parameters, $\mathcal{G}$ and $\Theta$ are the graph and parameter space respectively. The triple $\left(\Omega, \Sigma_{u}, B\right)$ are mathematical related. Suppose $X_{t} \sim \mathcal{N}\left(0, \Sigma_{x x}\right)$ and $Y_{t} \mid X_{t} \sim \mathcal{N}\left(B X_{t}, \Sigma_{u}\right), B$ and $\Sigma_{u}$ can be obtained from the covariance matrix of $D_{t}$ (i.e. $\Sigma=\Omega^{-1}$ ) by

$$
B=\Sigma_{y x} \Sigma_{x x}^{-1}, \quad \Sigma_{u}=\Sigma_{y y}-\Sigma_{y x} \Sigma_{x x}^{-1} \Sigma_{x y}
$$

where $\Sigma_{y x}$ is $n \times n p$ covariances between $Y_{t}$ and $X_{t}, \Sigma_{x x}$ is $n p \times n p$ covariances among $X_{t}$ and $\Sigma_{y y}$ is $n \times n$ covariances among $Y_{t}$. Given $B, \Sigma_{u}$ and $\Sigma_{x x}, \Omega$ can equally be obtained using the well-known Sherman-Morrison-Woodbury formula (Woodbury, 1950),

$$
\Omega=\Sigma^{-1}=\left(\begin{array}{cc}
\Sigma_{x x}^{-1}+B^{\prime} \Sigma_{u}^{-1} B & -B^{\prime} \Sigma_{u}^{-1} \\
-\Sigma_{u}^{-1} B & \Sigma_{u}^{-1}
\end{array}\right), \quad \text { where } \quad \Sigma=\left(\begin{array}{cc}
\Sigma_{x x} & \Sigma_{x y} \\
\Sigma_{y x} & \Sigma_{y y}
\end{array}\right)
$$

By defining $B=(G \circ \Phi)$, equation (4) shows how $\Omega$ relates to $G$ through $B$. The specification of the BG-VAR model is completed with the choice of a hierarchical prior on the lag order $p$, the graph structure $G$ and the parameter $\Omega$.

We now focus on the estimation procedure for the graph structure $(G)$ associated with the temporal dependence between the regional house prices. In the Bayesian framework, the joint prior distribution of $(p, G, \Omega)$ is given by $\operatorname{Pr}(p, G, \Omega)=\operatorname{Pr}(p) \operatorname{Pr}(G \mid p) \operatorname{Pr}(\Omega \mid p, G)$. It is important to first select the optimal lag order for the VAR model. Following Ahelegbey et al. (2016b), we choose $p$ in the range $0<p_{\min }<p_{\max }<\infty$, for some lower bound $p_{\text {min }}$ and upper bound $p_{\max }$. More specifically, we assume $p$ follows a discrete uniform prior on $\left\{p_{\min }, \ldots, p_{\max }\right\}$ with a distribution

$$
\operatorname{Pr}(p)=\frac{1}{p_{\max }-p_{\min }+1}
$$

Since we seek to estimate the regional market that is central in the spread of house price volatility from the data, it is more reasonable to assume a priori that any region is equally likely to play this role. This implies that the graph structure can be represented as a product of local sub-graphs of each equation of the model and may be written as

$$
\operatorname{Pr}(G \mid p)=\prod_{i=1}^{n} \operatorname{Pr}\left(\pi_{i} \mid p\right)
$$

where $\pi_{i}=\left\{j=1, \ldots, n p: G_{i j}=1\right\}$ is the set of price volatilities of the $i$-th equation predictors.

We formulate in what follows, the standard techniques for estimating $G$ also described by Ahelegbey et al. (2016a,b). We assume for each edge $G_{i j}$, an independent Bernoulli trial with 
conditional prior probability

$$
\operatorname{Pr}\left(\pi_{i} \mid p, \gamma\right)=\gamma^{\left|\pi_{i}\right|}(1-\gamma)^{n p-\left|\pi_{i}\right|}
$$

where $\left|\pi_{i}\right|$ is the cardinality of $\pi_{i}$ and $\gamma \in(0,1)$ is the Bernoulli parameter. We use a uniform graph prior by choosing $\gamma=0.5$ so that $\operatorname{Pr}\left(\pi_{i} \mid p, \gamma=0.5\right)=2^{-n p}$ and $\operatorname{Pr}(G \mid p) \propto 1$.

Following standard Bayesian paradigm, we also assume that $\Omega$ conditional on $p$ and a complete graph $G$ is Wishart distributed, $\Omega \sim \mathcal{W}\left(\nu, \underline{S}^{-1}\right)$, with density

$$
\operatorname{Pr}(\Omega \mid p, G)=\frac{1}{K_{d}(\nu, \underline{S})}|\Omega|^{\frac{(\nu-d-1)}{2}} \exp \left\{-\frac{1}{2}\langle\Omega, \underline{S}\rangle\right\}
$$

where $\langle A, B\rangle=\operatorname{tr}\left(A^{\prime} B\right)$ is the trace inner product, $\nu$ is the degree of freedom, $\underline{S}$ is the prior sum of squared matrix and $K_{d}(\nu, \underline{S})$ is the normalizing constant. The likelihood of a random sample $\mathcal{D}=\left(D_{1}, \ldots, D_{T}\right)$ is multivariate Gaussian with density

$$
\operatorname{Pr}(\mathcal{D} \mid p, \Omega, G)=(2 \pi)^{-\frac{1}{2} d T}|\Omega|^{\frac{1}{2} T} \exp \left\{-\frac{1}{2}\langle\Omega, \hat{S}\rangle\right\}
$$

where $\hat{S}=\sum_{t=1}^{T} D_{t} D_{t}^{\prime}$ is a $d \times d$ sample sum of squared matrix.

Given that $G$ is unknown, a standard Bayesian approach for determining the graph structure is to integrate out $\Omega$ from (9) with respect to its prior given by

$$
\operatorname{Pr}(\mathcal{D} \mid p, G)=\int \operatorname{Pr}(\mathcal{D} \mid p, \Omega, G) \operatorname{Pr}(\Omega \mid p, G) d \Omega=\frac{K_{d}(\nu+T, \underline{S}+\hat{S})}{(2 \pi)^{\frac{1}{2} d T} K_{d}(\nu, \underline{S})}
$$

where $\underline{S}+\hat{S}$ is the posterior sum of squared matrix. The expression (10) is the marginal likelihood function expressed as ratio of the normalising constants of the Wishart posterior and prior. Following standard application, the marginal likelihood factorises into the product of local terms, each involving $Y_{i, t}$ and its set of selected predictors, $X_{\pi_{i}, t}$, given by

$$
\operatorname{Pr}(\mathcal{D} \mid p, G)=\prod_{i=1}^{n} \operatorname{Pr}\left(\mathcal{D} \mid p, G_{i, \pi_{i}}\right)=\prod_{i=1}^{n} \frac{\operatorname{Pr}\left(\mathcal{D}^{\left(i, \pi_{i}\right)} \mid p, G\right)}{\operatorname{Pr}\left(\mathcal{D}^{\left(\pi_{i}\right)} \mid p, G\right)}
$$

where $\mathcal{D}^{\left(i, \pi_{i}\right)}$ and $\mathcal{D}^{\left(\pi_{i}\right)}$ are sub-matrices of $\mathcal{D}$ consisting of $\left(Y_{i, t}, X_{\pi_{i}, t}\right)$ and $X_{\pi_{i}, t}$ respectively. Let $w_{i} \in\left(\{i\} \cup \pi_{i}\right)$. The closed-form expression for the left-hand side of (11) is given by

$$
\operatorname{Pr}\left(\mathcal{D}^{w_{i}} \mid p, G\right)=\frac{\pi^{-\frac{1}{2} T\left|w_{i}\right|} \nu^{\frac{1}{2} \nu\left|w_{i}\right|}}{(\nu+T)^{\frac{1}{2}(\nu+T)\left|w_{i}\right|}} \frac{\left|\underline{\Sigma}_{w_{i}}\right| \frac{1}{2} \nu}{\left|\bar{\Sigma}_{w_{i}}\right|^{\frac{1}{2}(\nu+T)}} \prod_{s=1}^{\left|w_{i}\right|} \frac{\Gamma\left(\frac{\nu+T+1-s}{2}\right)}{\Gamma\left(\frac{\nu+1-s}{2}\right)}
$$

where $\left|w_{i}\right|$ is the cardinality of $w_{i}, \underline{\Sigma}_{w_{i}}$ and $\bar{\Sigma}_{w_{i}}$ are the prior and posterior covariance matrices of $\mathcal{D}^{w_{i}}$. Again, we follow standard practice and set $\underline{\Sigma}_{w_{i}}=I_{\left|w_{i}\right|}$, where $I_{\left|w_{i}\right|}$ is a $\left|w_{i}\right|$ dimensional 
identity matrix. ${ }^{2}$ By definition, (12) consists of a component that is independent of $\bar{\Sigma}_{w_{i}}$. We can reduce the computational time by expressing this independent component as a function $Q_{\nu}\left(\left|w_{i}\right|, p, T\right)$ given by

$$
Q_{\nu}\left(\left|w_{i}\right|, p, T\right)=\frac{\pi^{-\frac{1}{2} T\left|w_{i}\right|} \nu^{\frac{1}{2} \nu\left|w_{i}\right|}}{(\nu+T)^{\frac{1}{2}(\nu+T)\left|w_{i}\right|}} \prod_{s=1}^{\left|w_{i}\right|} \frac{\Gamma\left(\frac{\nu+T+1-s}{2}\right)}{\Gamma\left(\frac{\nu+1-s}{2}\right)}
$$

Since for each equation, we have $n p$ number of explanatory variables, $\left|w_{i}\right|$ will be bounded below by 1 and above by $n p+1$. Thus, we can set $\nu=n p+2$. Given $\nu, T$ and $p, Q_{\nu}\left(\left|w_{i}\right|, p, T\right)$ does not directly depend on the variables in $w_{i}$ but on $\left|w_{i}\right| \in\{1, \ldots, n p+1\}$. Hence, (12) may be expressed as

$$
\operatorname{Pr}\left(\mathcal{D}^{w_{i}} \mid p, G\right)=Q_{\nu}\left(\left|w_{i}\right|, p, T\right)\left|\bar{\Sigma}_{w_{i}}\right|^{-\frac{1}{2}(\nu+T)}
$$

The posterior covariance matrix of $\mathcal{D}$ is also given by

$$
\bar{\Sigma}=\frac{1}{\nu+T}\left(\nu I_{d}+\sum_{t=1}^{T} D_{t} D_{t}^{\prime}\right)
$$

Thus, $\bar{\Sigma}_{w_{i}}$ in (14) can be obtained as a sub-matrix of $\bar{\Sigma}$ which corresponds to the elements in $w_{i}$. Pre-computing $\bar{\Sigma}$ and $Q_{\nu}\left(\left|w_{i}\right|, p, T\right)$ for $\left|w_{i}\right|$ given $\nu, T$ and $p$, before sampling the network matrix reduces the computational complexity and makes the algorithm efficient. The details of sampling the network structure is provided in Appendix A.

\section{Description of Data}

This paper studies the temporal spatial dependence and diffusion pattern of house prices in the Netherlands. In this section, we give a brief background to the regional housing market in the Netherlands and describe the data. The spatial units for our analysis are the twelve official Dutch provinces ${ }^{3}$ and these include: Drenthe, Flevoland, Friesland, Gelderland, Groningen, Limburg, Noord-Brabant, Noord-Holland, Overijssel, Zuid-Holland, Utrecht and Zeeland (see map in Figure 2). Zuid-Holland is the largest in terms of GDP (141.758 billion Euros in 2014), followed by Noord-Holland (133.358 billion Euros in 2014). Zeeland is the smallest with estimated GDP of 11.429 billion Euros in 2014 according to Statistic Netherlands. The capital Amsterdam is hosted by Noord-Holland while the government seat (The Hague) is located in Zuid-Holland.

Following result in the extant literature that house price shocks from the "mega economic districts" diffuse through to the peripheral regions (see Gong et al., 2016; Holly et al., 2011),

\footnotetext{
${ }^{2}$ For any $n \times n$ identity matrix $A$, we have $|A|=1$.

${ }^{3}$ In this paper, we use region and province interchangeably.
} 
our initial expectation is that Noord-Holland or Zuid-Holland at certain periods may be found central in the house price diffusion mechanism in the Netherlands.

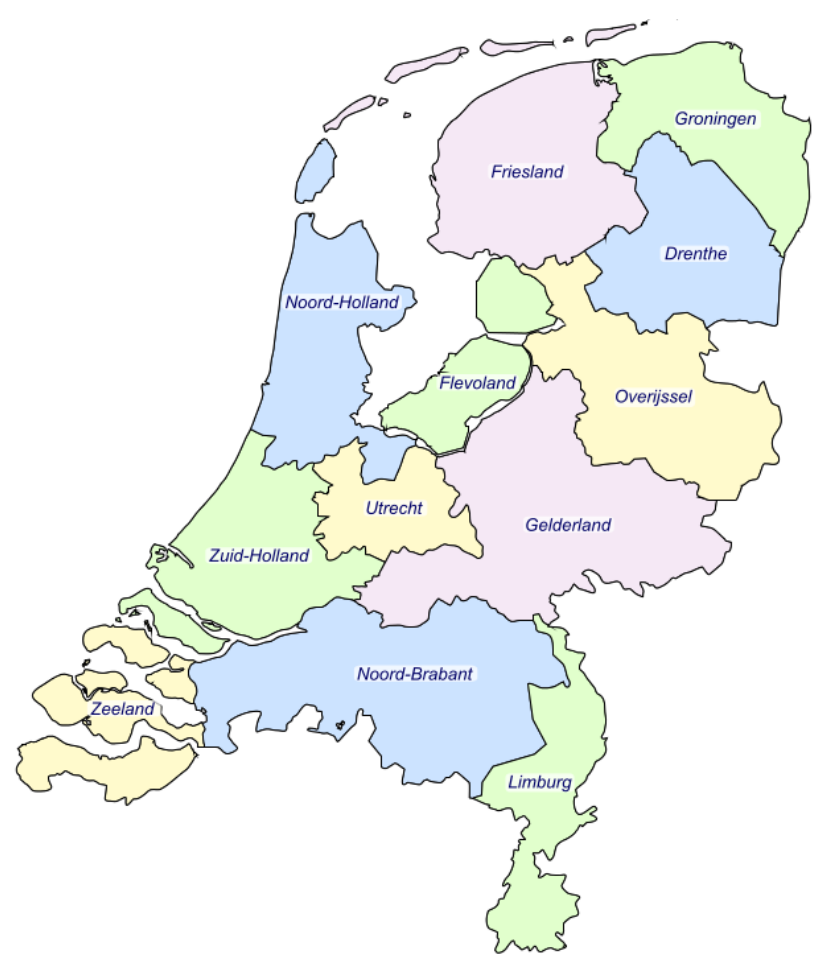

Figure 2: The twelve provinces of the Netherlands. Source: d-maps.com

We defined the temporal volatilities from quarterly house price indexes between 1995Q1 and 2016Q1 for owner-occupied dwellings in the analysis of this paper. The original house price indexes were obtained from the Centraal Bureau voor de Statistiek (CBS). CBS is the Dutch official statistical agency which publishes, among others, housing statistics online. A simple plot of the house price indexes (Figure 3) shows a common trend in the growth of house prices in all the twelve regional markets before and after the GFC. The periods prior to 2009 show relatively volatile and faster house price appreciation which may be attributed to many factors. Most importantly, however, the Dutch government had forcefully promoted home ownership during these periods with the National Mortgage Guarantee scheme and through an income tax structure that offered generous rebates on the payment of mortgage interest (see, Boelhouwer et al., 2004; Boelhouwer, 2002; Elsinga, 2003; Toussaint and Elsinga, 2007). These incentive packages generally made it cheaper for individual households to purchase their own dwellings. Consequently, there was an increase demand which also led to an upward and more volatile house prices before the crisis.

As in other countries, financial institutions in the Netherlands were also hit by the 2007-08 GFC. The impact of the crisis on house prices however started in the last quarter of 2008 as seen in Figure 3. Following the GFC, average house prices in the Netherlands declined by 


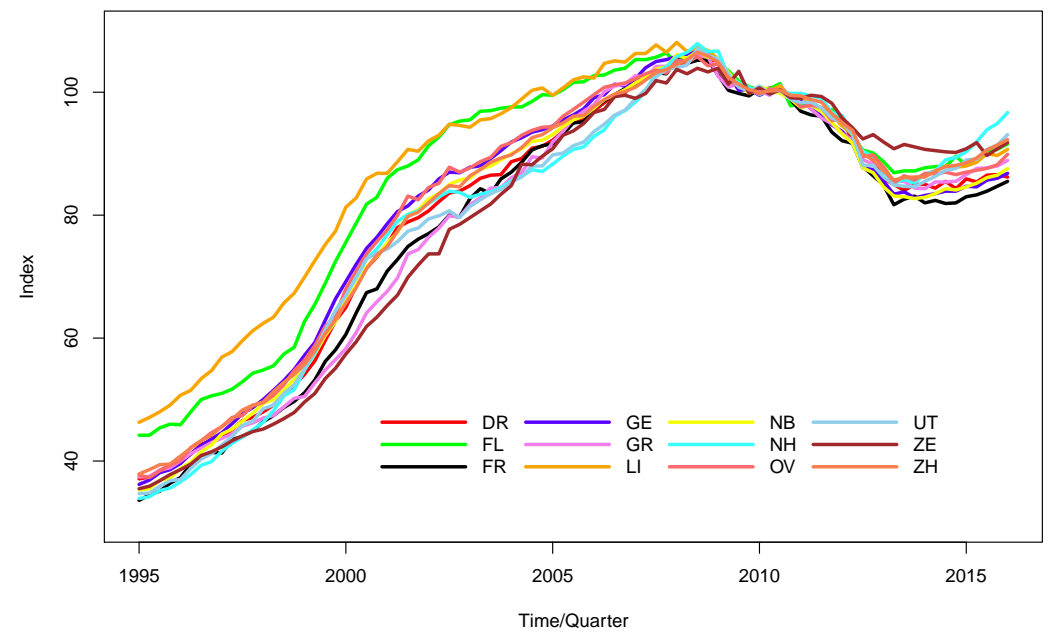

Figure 3: CBS' house price index for the 12 Dutch provinces. Note: $D R=$ Drenthe, $F L=$ Flevoland, $F R=$ Friesland, $G E=$ Gelderland, $G R=$ Groningen, $L I=\operatorname{Limburg}, N B=$ Noord-Brabant,$N H=$ Noord-Holland, $O V=$ Overijssel, $U T=$ Utrecht, $Z E=$ Zeeland, $Z H=$ Zuid-Holland.

almost 25\% between 2009 and 2013. Teulings (2014), attributed the collapse in the Dutch property values with the higher unemployment and redundancy rates during the meltdown. Other scholars however blamed the collapse on the Dutch financial institutions who tightened up mortgage accessibility and impeded new home buyers from the market (Boelhouwer, 2014; Bardhan et al., 2011). Since the beginning of 2014, there have been gradual recovery of Dutch house prices, somewhat faster in Zuid-Holland and Noord-Holland.

\section{Spatial and temporal house price dynamics}

In studying temporal house price diffusion, we are interested here in understanding if temporal house price volatilities in one region of the Netherlands is a result of earlier shock(s) to house prices in other regions. Our methodology adopts the the network approach. We followed Martens and Van Dijk (2007) to define the house price volatilities for each region as the squared returns given by

$$
S R_{t}=\left[100\left(\log I_{t}-\log I_{t-1}\right)\right]^{2}
$$

where $I_{t}$ is the house price index at the time $t$. Figure 4 summarises the temporal regional house price volatilities.

We began by estimating the temporal interdependencies between the regional house price volatilities from the network structure using a twenty-quarter rolling window. This was to examine the degree of temporal interdependencies in the regional house prices and to discern the patterns over time. Figure 5 presents the network density associated with the volatility interdependencies. The network density is a crude measure which indicates the proportion 


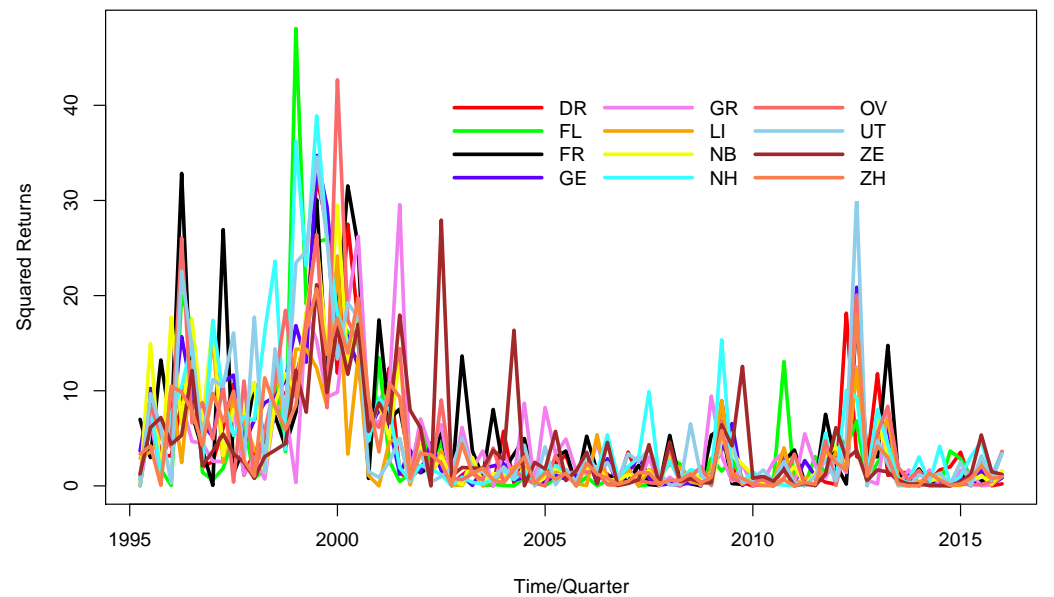

Figure 4: Regional house price volatilities. Note: $D R=$ Drenthe, $F L=$ Flevoland, $F R=$ Friesland, $G E=$ Gelderland, $G R=$ Groningen, $L I=$ Limburg, $N B=$ Noord-Brabant, $N H=$ Noord-Holland, $O V=$ Overijssel, $U T=$ Utrecht, $Z E=$ Zeeland, $Z H=$ Zuid-Holland.

of regions whose temporal house price volatilities influence (or are caused by earlier) price movements in other regions. Over the study period, the figure shows that the network density increased from 1995 to 2003, then decreased until 2008, after which it began to increase again.

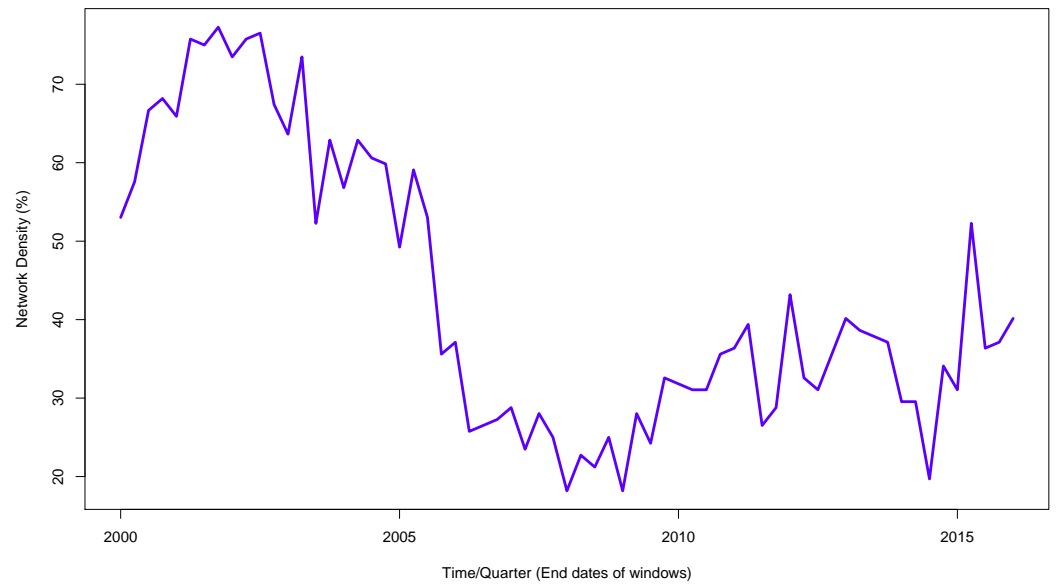

Figure 5: Density of networks of house price volatility in the 12 Dutch regional housing markets estimated over the period 1995Q2 - 2016Q1 using a rolling window of 20 quarters.

Interestingly, the above sub-periods somehow coincide with recognisable stages in the development of house prices in the Netherlands. For instance, most Dutch researchers recognise the period 1995-2003 as one during which house prices increased legitimately because of rise in disposable income and government stimulation of the housing market (Boelhouwer 


\begin{tabular}{lcccc}
\hline & Links & Density & Average Degree & $\begin{array}{c}\text { Average Path } \\
\text { Length }\end{array}$ \\
\hline $1995-2003$ & 106.00 & 0.80 & 17.67 & 1.20 \\
$2003-2008$ & 30.00 & 0.23 & 5.00 & 2.23 \\
$2008-2016$ & 43.00 & 0.33 & 7.17 & 1.65 \\
\hline
\end{tabular}

Table 1: The network statistics for the three graphs. Connected nodes have graph distance 1.

\begin{tabular}{lccccccccccccc}
\hline Period & DR & FL & FR & GE & GR & LI & NB & NH & OV & UT & ZE & ZH \\
\hline $1995-2003$ & 4 & 2 & 4 & 4 & 3 & 1 & 1 & 2 & 2 & 4 & 4 & 2 \\
$2003-2008$ & 4 & 1 & 1 & 1 & 1 & 1 & 1 & 1 & 1 & 1 & 1 \\
$2008-2016$ & 1 & 1 & 1 & 1 & 1 & 1 & 1 & 1 & 1 & 1 & 1 & 1 \\
\hline
\end{tabular}

Table 2: Equation-specific lag order of each equation for the sub-periods. Note: $D R=$ Drenthe, $F L$ $=$ Flevoland, $F R=$ Friesland, $G E=$ Gelderland, $G R=$ Groningen, $L I=$ Limburg, $N B=$ NoordBrabant, $N H=$ Noord-Holland, $O V=$ Overijssel, $U T=$ Utrecht, $Z E=$ Zeeland, $Z H=$ Zuid-Holland.

et al., 2004; Boelhouwer, 2002; De Vries, 2010; Toussaint and Elsinga, 2007). Moreover, some analysts argue that the Dutch house price development from 2003-2008 was mostly due to over-valuation and speculative investment activities which also precipitated the crisis that started from the last quarter of 2008 (Xu-Doeve, 2010; Aalbers, 2009a,b).

By reasoning from the perspective of Meen (1999), it is conceivable that the higher regional house price volatility interdependencies from 1995 to 2003 might be, among other things, due to some common shocks or the correlated fundamentals. As pointed out earlier, the Dutch home ownership sector underwent reforms from the mid-1990s to early part of the 2000s where the government policy regulations and consequently mortgage interest rates became somewhat determinate fundamentals of house price development throughout the Netherlands. The varied response rate of house prices to these fundamentals in the different regions as explained by (Meen, 1996, 1999), may play a role in the network volatility interdependencies displayed from 1995 to 2003 . On the other hand, the period after 2003 when it is also widely believed that there were speculative investment activities, it is more likely that the temporal house price dependence then, might be due to spatial arbitrage or equity transfer. This is in line with the arguments put forward by Meen (1999).

We turned the focus on the three sub-periods: 1995Q1-2003Q4, 2003Q1-2008Q4 and 2008Q1-2016Q1 to throw more light on the interdependence between the regional house price volatilities. The summary statistics and optimal lag order associated with the network structure for each specific sub-period are presented in Tables 1 and 2. The average path length for example, represents the average graph-distance between all pair of nodes, where interconnected nodes have graph distance of 1 . In general, the higher the graph distance the slower it takes house price shocks in one region to cascade systemically. Table 1 also indicates the total links and average degree which are important for the network analysis.

The interest here is to identify the regions with temporal house price volatilities that are predominately causal and their specific interconnection with the others. These regions are 
important because they play key role in the transmission of house price shocks. If identified, it helps policy makers to monitor the housing market and to direct regulations that prevent systemic failure. In the network terminology, these regions are the hub-centralities (see, Benzi et al., 2013). The network structures for the three sub-periods are presented in Figure 6. As in in Figure 5, these network structures show the degree to which the regional house price volatilities are interdependent or connected to one another in each sub-period.

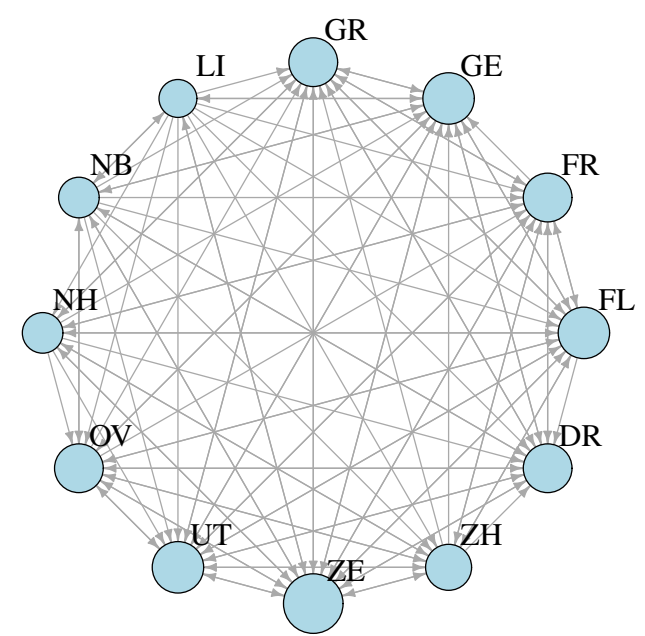

(a) $1995-2003$

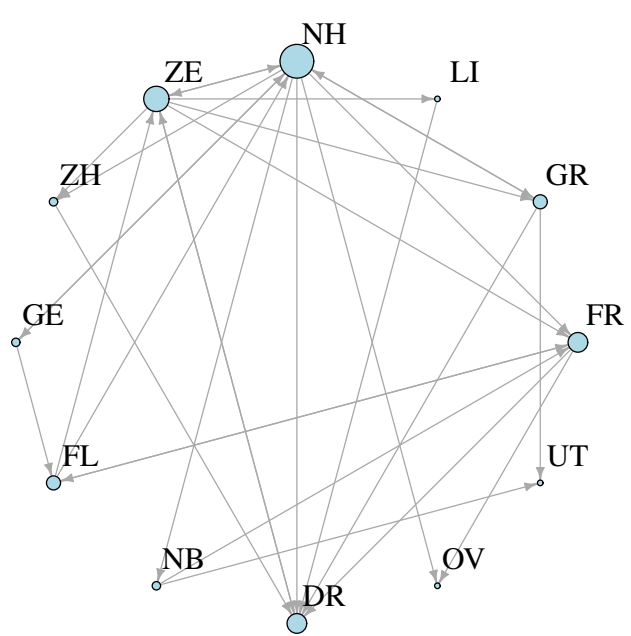

(b) $2003-2008$

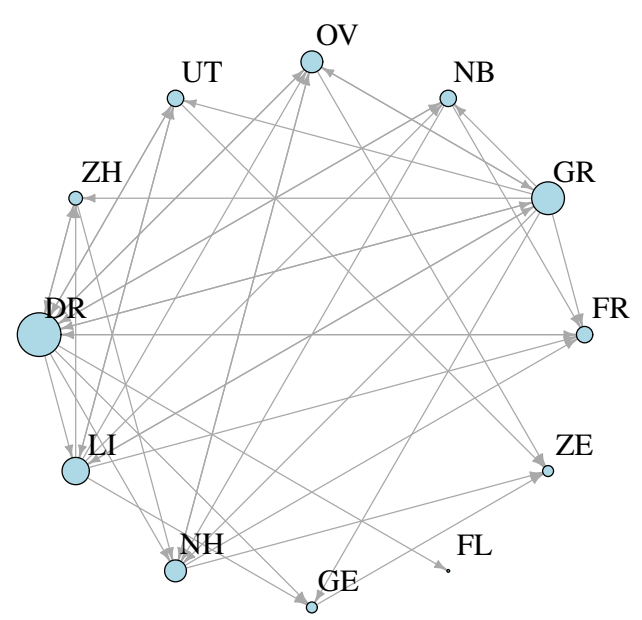

(c) $2008-2016$

Figure 6: Network diagrams of temporal volatility connectedness in the regional market among the 12 Dutch provinces during (6a) 1995 - 2003, (6b) 2003 - 2008, and (6c) $2008-2016$. The size of the nodes is proportional to their degree (in-degree + out-degree). Note: $D R=$ Drenthe, $F L=$ Flevoland, $F R=$ Friesland, $G E=$ Gelderland, $G R=$ Groningen, $L I=$ Limburg, $N B=$ Noord-Brabant, $N H=$ Noord-Holland, $O V=$ Overijssel, $U T=$ Utrecht, $Z E=$ Zeeland, $Z H=$ Zuid-Holland.

We determined the hub-centrality for each sub-period using the Katz measure (Katz, 1953). Table 3 presents the Katz centrality measure and the ranks associated with the network structure in Figure 6 for each region. The table show that Limburg ranked the most central in 


\begin{tabular}{lccc|ccc|ccc}
\hline & \multicolumn{3}{c}{$1995-2003$} & & \multicolumn{3}{c}{$2003-2008$} & \multicolumn{3}{c}{$2008-2016$} \\
\hline & Cent. & Rank & Dist. & Cent. & Rank & Dist. & Cent. & Rank & Dist \\
\hline Drenthe & 284.68 & 12 & 1 & 1.66 & 10 & 1 & $\mathbf{2 8 . 0 2}$ & 1 \\
Flevoland & 481.32 & 6 & 1 & 3.78 & 3 & 2 & 1.00 & 11 & 1 \\
Friesland & 387.45 & 9 & 1 & 3.55 & 5 & 1 & 1.89 & 9 & 1 \\
Gelderland & 310.65 & 10 & 1 & 2.42 & 7 & 1 & 1.71 & 10 & 1 \\
Groningen & 302.52 & 11 & 1 & 3.70 & 4 & 1 & 25.67 & 2 & 1 \\
Limburg & $\mathbf{6 5 6 . 8 0}$ & 1 & 0 & 1.86 & 8 & 2 & 17.77 & 3 & 1 \\
Noord-Brabant & 559.68 & 3 & 1 & 2.45 & 6 & 1 & 5.35 & 5 & 1 \\
Noord-Holland & 524.50 & 4 & 1 & $\mathbf{1 1 . 0 6}$ & 1 & 0 & 4.58 & 6 & 1 \\
Overijssel & 478.83 & 7 & 1 & 1.00 & 12 & 1 & 6.06 & 4 & 1 \\
Utrecht & 408.87 & 8 & 1 & 1.00 & 11 & 2 & 4.11 & 7 & 1 \\
Zeeland & 563.77 & 2 & 1 & 8.24 & 2 & 1 & 1.00 & 12 \\
Zuid-Holland & 499.19 & 5 & 1 & 1.86 & 9 & 1 & 3.38 & 8 \\
\hline
\end{tabular}

Table 3: Hub centrality among the Dutch major provinces during the sub-periods. Note: Cent. means hub centrality, Dist. measures distance from hub to other markets. Connected nodes have graph distance 1. Bold values indicate hubs.

the network of regional house price volatility for the Netherlands in the sub-period, 1995-2003. Between 2003-2008, Noord-Holland is ranked the most central while Drenthe is ranked the most central for the sub-period 2008-2016. The Table 3 also indicates the network distance which may also be used to capture the diffusion dynamics of temporal house price volatilities from these central regions.

The network distance is by definition the length of the shortest path between two nodes in the network. A network distance of 1 denotes a direct interconnection while a distance of 2 indicates the interdependence between two nodes, mediated by another node. In tandem with this description, the results of Table 3 may be interpreted to mean that, temporal house price volatility from Limburg in the period 1995-2003 had a causal influence on the volatility of house prices in the other regions. Similarly, we find that temporal house price volatility in Noord-Holland had directly affected other regions between 2003-2008, except for Flevoland, Limburg and Utrecht where this was mediated. Also for the period 2008-2016, the table shows volatility of house prices from Drenthe directly impacted house price movements in all regions apart from Zeeland where it was mediated. Furthermore, the optimal lag order of 1 selected as in Table 2 reveals that temporal house price volatilities from these central regions in any quarter caused a temporal house price volatility in other regions in the following quarter.

\section{Discussion and concluding remarks}

In an effort to revive the housing markets that have collapsed in many countries following the 2007-2008 Global Financial Crisis (GFC), there is an ongoing research agenda that seeks understanding into the spatio-temporal dynamics of house prices. This paper makes three main contributions to this new research area. Firstly, the paper studied the spatio-temporal house price dynamics in the unique context of the Netherlands, which is first of its kind. Here, the paper specifically asked if there is temporal spatial dependence of house prices in 
the Netherlands. It then investigated the diffusion pattern and identified the specific regions where temporal house price volatilities are likely to spread.

For the second contribution, the paper demonstrated the usefulness of graphical and network techniques in analysing the spatio-temporal house price dynamics. Particularly the paper adopted the newly proposed Bayesian graphical vector autoregression (BG-VAR) model which is in general more efficient in identifying dependence patterns between multiple variables than the traditional concept of Granger causality. As a third contribution, the paper proposed a simple data driven techniques to identify the regional housing sub-market where diffusion of temporal house price volatilities may predominately start.

In the empirical analysis, the paper used temporal volatilities constructed from quarterly house price indexes for owner-occupied dwelling between 1995Q1 and 2016Q1. The results, based on the BG-VAR model and various network statistics, support a temporal dependence of the regional house prices in the Netherlands. Nonetheless, we observed a varying degree of this temporal interdependence over the study period. We found that the Dutch regional house prices were highly interdependent between 1995 and 2003. After 2003, the degree of interdependence weakened until 2008 and again increase from 2008 to 2016 . Recognising that this periods coincide with historic stages in the development of house prices in the Netherlands, we studied in details the spatio-temporal dynamics in the sub-periods: 1995-2003, 2003-2008 and 2008-2016.

During these sub-periods: 1995-2003, 2003-2008 and 2008-2016, we identified Limburg, Noord-Holland and Drethe as the respective regional housing markets that are most central in the diffusion of temporal house price volatility. One lesson from this finding is that contrary to the extant literature (e.g. Meen, 1999; Holly et al., 2011; Gong et al., 2016) which posit that temporal house price volatility spread from some economically "mega city", there is also the possibility that the diffusion may equally start from an "economically smaller" region (like Drenthe in the Dutch case under study here). Moreover, the results of the paper suggest that the central region where the house price diffusion may predominantly starts is not constant, instead it changes over time depending on the economic conditions. For instance, while many Dutch researchers recognise the sub-period 1995-2003 as an era when house prices increased proportionately to the fundamentals, the sub-period 2003-2008 is identified with the era preceding the GFC when its also believed that residential properties were over-valued and there were speculative investment activities. In each of these sub-periods, we found different central regions for the house price diffusion.

Previous literature also suggest that temporal house price volatility diffuse from the central region and slowly through to the remote peripheral areas. We analyse this diffusion pattern in this paper with the network distance. The network distance yields literally the number of regions to which temporal house price volatilities may diffuse having started from the central region. This however augments the graphical aids provided by the results of the BG-VAR 
detailed in the main text. For the Netherlands, we identified that the diffusion trajectory is limited to at most 2 regions, following a maximum network distance of 1,2,2 in the respective sub-periods studied.

In application, the results in this paper are relevant for policy makers who wish to stimulate segments of the housing market or to direct regulations that is aimed at avoiding temporal regional house price volatilities from cascading systemically to other regions. In particular, because house prices are temporally interdependent, stimulating a segment of the market may lead to a ripple effect on the other sub-markets. Hence, policy makers may be guided by results of this paper to formulate bundle of regulations aimed at reviving the housing market in a collective manner. On the other hand, by identifying in a current period one region where temporal house price volatilities may largely diffuse, policy makers may carefully examine the house price developments in that sub-market and address any abnormal price increases before the spillover effect becomes widespread. Investors may equally find the results of this paper applicable in diversifying and managing risks of their housing portfolios.

\section{Acknowledgements}

We thank Professor Dr. Marja Elsinga and Professor Dr. Jan de Haan for carefully reading the manuscript and providing very helpful suggestions. The authors are also grateful to Yunlong Gong, Qi Tu and Job Gbadegesin for their useful comments on earlier versions of this paper. Authors' research is supported by funding from Statistics Netherlands (CBS) and the US Air Force Office for Scientific Research (AFOSR), whose grant number is FA9550-12-1-0102.

\section{References}

Aalbers M. 2009a. Geographies of the Financial Crisis. Area 41: 34-42.

Aalbers MB. 2009b. The Sociology and Geography of Mortgage Markets: Reflections on the Financial Crisis. International Journal of Urban and Regional Research 33: 281-290.

Ahelegbey DF. 2016. The Econometrics of Bayesian Graphical Models: A Review With Financial Application. Journal of Network Theory in Finance 2: 1-33.

Ahelegbey DF, Billio M, Casarin R. 2016a. Bayesian Graphical Models for Structural Vector Autoregressive Processes. Journal of Applied Econometrics 31: 357-386.

Ahelegbey DF, Billio M, Casarin R. 2016b. Sparse graphical vector autoregression: A bayesian approach. Annals of Economics and Statistics/Annales d'Économie et de Statistique : 333-361.

Balcilar M, Beyene A, Gupta R, Seleteng M. 2013. Ripple Effects in South African House Prices. Urban Studies 50: 876-894.

Bardhan A, Edelstein R, Kroll C. 2011. Global Housing Markets: Crises, Policies and Institutions, volume 17. John Wiley \& Sons.

Benzi M, Estrada E, Klymko C. 2013. Ranking Hubs and Authorities Using Matrix Functions. Linear Algebra and its Applications 438: 2447-2474.

Boelhouwer P. 2014. Financial Institutions have Owner-occupancy in a Stranglehold: An Explanation for the Recession in the Dutch Housing Market. Technical report, Delft University of Technology, OTB Research Institute for the Built Environment. 
Boelhouwer P, Haffner M, Neuteboom P, Vries P. 2004. House Prices and Income Tax in the Netherlands: An International Perspective. Housing Studies 19: 415-432.

Boelhouwer PJ. 2002. Capital Accumulation via Homeownership: The Case of the Netherlands. European Journal of Housing Policy 2: 167-181.

Brady RR. 2014. The Spatial Diffusion of Regional Housing Prices across US States. Regional Science and Urban Economics 46: 150-166.

Canarella G, Miller S, Pollard S. 2012. Unit Roots and Structural Change an Application to US House Price Indices. Urban Studies 49: 757-776.

Cook S. 2003. The Convergence of Regional House Prices in the UK. Urban Studies 40: 2285-2294.

Cook S. 2005. Detecting Long-run Relationships in Regional House Prices in the UK. International Review of Applied Economics 19: 107-118.

Cotter J, Gabriel SA, Roll R. 2011. Integration and Contagion in US Housing Markets. Working paper, Social Science Research Network.

De Vries P. 2010. Measuring and Explaining House Price Developments, volume 36. IOS Press.

Dickey DA, Fuller WA. 1979. Distribution of the Estimators for Autoregressive Time Series with a Unit Root. Journal of the American Statistical Association 74: 427-431.

Eichler M. 2007. Granger Causality and Path Diagrams for Multivariate Time Series. Journal of Econometrics 137: $334-353$.

Elsinga M. 2003. Encouraging Low Income Home Ownership in the Netherlands; Policy Aims, Policy Instruments and Results. In ENHR-conference.

Giussani B, Hadjimatheou G. 1991. Modeling Regional House Prices in the United Kingdom. Papers in Regional Science 70: 201-219.

Gong Y, Hu J, Boelhouwer PJ. 2016. Spatial Interrelations of Chinese Housing Markets: Spatial Causality, Convergence and Diffusion. Regional Science and Urban Economics 59: 103-117.

Gupta R, Miller SM. 2012a. "Ripple Effects" and Forecasting Home Prices in Los Angeles, Las Vegas, and Phoenix. The Annals of Regional Science 48: 763-782.

Gupta R, Miller SM. 2012b. The Time-series Properties of House Prices: A Case Study of the Southern California Market. The Journal of Real Estate Finance and Economics 44: 339-361.

Holly S, Pesaran MH, Yamagata T. 2010. A Spatio-temporal Model of House Prices in the USA. Journal of Econometrics 158: 160-173.

Holly S, Pesaran MH, Yamagata T. 2011. The Spatial and Temporal Diffusion of House Prices in the UK. Journal of Urban Economics 69: 2-23.

Holmes MJ. 2007. How Convergent are Regional House Prices in the United Kingdom? Some New Evidence from Panel Data Unit Root Testing. Journal of Economic and Social Research 9: 1-17.

Holmes MJ, Grimes A. 2008. Is There Long-run Convergence Among Regional House Prices in the UK? Urban Studies 45: 1531-1544.

Katz L. 1953. A New Status Index Derived from Sociometric Analysis. Psychometrika 18: 39-43.

Lauritzen SL. 1996. Graphical Models. Oxford University Press, Oxford.

Lee CC, Chien MS. 2011. Empirical Modelling of Regional House Prices and the Ripple Effect. Urban Studies 48: 2029-2047.

Martens M, Van Dijk D. 2007. Measuring Volatility with the Realized Range. Journal of Econometrics 138: $181-207$.

Meen G. 1996. Spatial Aggregation, Spatial Dependence and Predictability in the UK Housing Market. Housing Studies 11: 345-372.

Meen G. 1999. Regional House Prices and the Ripple Effect: A New Interpretation. Housing Studies 14: 733-753.

Nanda A, Yeh JH. 2014. Spatio-temporal Diffusion of Residential Land Prices Across Taipei Regions. Springer- 
Plus 3: 1.

Pollakowski HO, Ray TS. 1997. Housing Price Diffusion Patterns at Different Aggregation Levels: An Examination of Housing Market Efficiency. Journal of Housing Research 8: 107.

Teulings CN. 2014. Unemployment and House Price Crises: Lessons for Fiscal Policy from the Dutch Recession. IZA Journal of European Labor Studies 3: 20.

Toussaint J, Elsinga M. 2007. The Netherlands: Positive Prospects and Equity Galore. In Home Ownership Beyond Asset and Security: Perceptions of Housing Related Security and Insecurity in Eight European Counties (Housing and Urban Policy Studies), volume 32. 287-313.

Vansteenkiste I, Hiebert P. 2011. Do House Price Developments Spill Over Across Euro Area Countries? Evidence from a Global VAR. Journal of Housing Economics 20: 299-314.

Vermeulen W, Rouwendal J. 2007. Housing Supply and Land Use Regulation in the Netherlands. Discussion paper, Tinbergen Institute.

Woodbury MA. 1950. Inverting Modified Matrices. Memorandum Report 42: 106.

Xu-Doeve WL. 2010. The Overvalued Housing Market in the Netherlands: A Conspiracy of Silence. In 5th Conference of the IFC, held. 25-26. 


\section{Appendix A. Sampling Network Structure}

The sampling of the graph structure in this paper follows the procedure described by Ahelegbey et al. (2016b). The method is summarised here for completeness. First, for a given lag order $p$, the initialisation of the Markov chain Monte Carlo (MCMC) is ran in two steps.

(i) Set $G^{0}$ to $n \times n p$ null matrix. This is the case when each equation has no predictor(s).

(ii) For each equation $i=1, \ldots, n$; test each $X_{j, t} \in X_{t}, j=1, \ldots, n p$ as a potential predictor of $Y_{i, t}$. If $\operatorname{Pr}\left(Y_{i, t} \mid X_{j, t}, p\right)>\operatorname{Pr}\left(Y_{i, t} \mid p\right)$, then set $G_{i, j}^{0}=1$, otherwise $G_{i, j}^{0}=0$.

These steps provide a good starting point for implementing the algorithm for sampling the network structure. The authors suggest to use the Gibbs sampling algorithm which proceeds at each $m$-th iteration as follows:

(i) Denote with $G^{(m-1)}$, the current network matrix and find $\pi_{i}^{(m-1)}$, the set of indexes of the non-zero elements of the $i$-th row of $G^{(m-1)}$.

(ii) Find $X_{\pi_{i}, t}^{(m-1)}$, the vector of elements in $X_{t}$ whose indexes corresponds to $\pi_{i}^{(m-1)}$.

(iii) Draw an index $k$ from the set of indexes of possible predictors, say $X_{k, t} \in X_{t}$.

(iv) Set $G^{*}=G^{(m-1)}$ and add/remove edge between $Y_{i, t}$ and $X_{k, t}$, i.e., $G_{i k}^{(*)}=1-G_{i k}^{(m-1)}$.

(v) Find $\pi_{i}^{(*)}$, the set of indexes of the non-zero elements of the $i$-th row of $G^{(*)}$ and $X_{\pi_{i}, t}^{(*)}$, the vector of elements in $X_{t}$ whose indexes corresponds to $\pi_{i}^{(*)}$.

(vi) Compute $\operatorname{Pr}\left(Y_{i, t} \mid X_{\pi_{i}, t}^{(m-1)}, p\right)$ and $\operatorname{Pr}\left(Y_{i, t}\left|X_{\pi_{i}, t}^{(*)}\right| p\right)$, and $R_{\alpha}=\frac{\operatorname{Pr}\left(Y_{i, t} \mid X_{\pi_{i}, t}^{(*)}, p\right)}{\operatorname{Pr}\left(Y_{i, t} \mid X_{\pi_{i}, t}^{(m-1)}, p\right)}$.

(vii) Sample $u \sim \mathcal{U}_{[0,1]}$ from a uniform distribution. If $u<\min \left\{1, R_{\alpha}\right\}$, set $G^{(m)}=G^{(*)}$, otherwise set $G^{(m)}=G^{(m-1)}$.

The above steps are implemented for a total of $M$ iterations and averaged over the sampled graphs. The posterior probability of an edge is then estimated by $\hat{e}_{i j}=\frac{1}{M} \sum_{m=1}^{M} G_{i j}^{(m)}$, where $G_{i j}^{(m)}$ is the edge from $X_{j, t}$ to $Y_{i, t}$ in the network matrix $G$ at the $m$-th iteration. See Ahelegbey et al. (2016a) for details on the convergence diagnostics of the MCMC chain. For simplicity, we estimate $\hat{G}_{i j}$ such that $\hat{G}_{i j}=1$, if $\hat{e}_{i j}>0.5$, and zero otherwise.

We construct a temporal network structure by transforming the estimate matrix $\hat{G}$ to an adjacency (square binary) matrx of a directed graph. Following the labeling of our network matrix as shown in Figure 1, the edges in the adjacency matrix indicate a direct link from a column label to a row label. For example $A_{i j}=1$ means $Y_{j} \rightarrow Y_{i}$. Let $A$ be an $n \times n$ null matrix. We construct the adjacency matrix following the steps below.

(i) For $i \neq j=1, \ldots, n$, denote with $y_{j}$, the set of indexes of $Y_{j, t-1}, \ldots, Y_{j, t-p} \in X_{t}$

(ii) Find $V_{i, y_{j}}=\hat{G}_{i, v_{j}}$, the vector of edges on the $i$-th row and the $y_{j}$ columns of $\hat{G}$

(iii) If $\sum V_{i, y_{j}} \neq 0$ then set $A_{i j}=1$, otherwise $A_{i j}=0$

The main diagonal of $A$ are therefore represented by zeros. The above is similar to testing, $H_{0}: B_{1, i j}=\ldots=B_{p, i j}=0$ against $H_{A}:$ Not $H_{0}, \forall i, j=\{1, \ldots, n\}, i \neq j$. 